\title{
INNER FUNCTIONS AND THE MAXIMAL IDEAL SPACE OF $H^{\infty}\left(U^{n}\right)$
}

\author{
S. H. KON
}

\begin{abstract}
For the case of the polydisc, Range has shown that the Shilov boundary $\partial_{n}$ of $H^{\infty}\left(U^{n}\right)$ is a proper subset of $\tau X_{n}$, the set of all restrictions of complex homomorphisms of $L^{\infty}\left(T^{n}\right)$ to $H^{\infty}\left(U^{n}\right)$. In this paper, we show that $\tau X_{n}$ is a proper subset of those complex homomorphisms of $H^{\infty}\left(U^{n}\right)$ which are unimodular on the class of all inner functions.
\end{abstract}

1. Introduction. Let $T^{n}$ be the distinguished boundary of the unit polydisc $U^{n}$ and denote the class of all bounded analytic functions on $U^{n}$ by $H^{\infty}\left(U^{n}\right)$. A function $f$ in $H^{\infty}\left(U^{n}\right)$ is said to be inner if its radial boundary values

$$
f^{*}(w)=\lim _{r \rightarrow 1} f(r w)
$$

are of modulus one almost everywhere (a.e.) on $T^{n}$ with respect to the normalised Lebesgue measure $m_{n}$ on $T^{n}$. The class of all inner functions on $U^{n}$ is denoted by $\Sigma_{n}$. Let

$$
H^{\infty} / \Sigma_{n}=\left\{f^{*} / I^{*}: f \in H^{\infty}\left(U^{n}\right), I \in \Sigma_{n}\right\},
$$

then its closure $\left[H^{\infty} / \Sigma_{n}\right]$ is the closed subalgebra generated by $H^{\infty} / \Sigma_{n}$ in $L^{\infty}\left(T^{n}\right)$. For the case of the unit disc $U$, the index $n=1$ will be omitted from all our notations.

Douglas and Rudin [1] have shown that $\left[H^{\infty} / \Sigma_{n}\right]=L^{\infty}(T)$, the main result here shows that this is no longer true for $n>1$.

2. A proper subalgebra of $L^{\infty}\left(T^{n}\right)$. First, a result of Rudin is needed, it will be stated with some additional details and estimates from the proof in [3, Theorem 5.4.8].

THEOREM 1. Let $A$ be a totally disconnected, compact subset of $T$ with $m(A)>0$. Defining $E_{1}=\left\{\left(w_{1}, w_{2}\right) \in T^{2}: w_{2} / w_{1} \in A\right\}, E_{1}$ is a compact, circular subset of $T^{2}$ with $m_{2}\left(E_{1}\right)>0$. Then there exists $F_{1}$ in $H^{\infty}\left(U^{2}\right)$ such that

(i) $3 / 5>\left|F_{1}^{*}\right|>2 / 5$ a.e. on $E_{1}$,

(ii) $11 / 10>\left|F_{1}^{*}\right|>9 / 10$ a.e. on $T^{2} \backslash E_{1}$.

For $n \geqslant 2$, we define $F \in H^{\infty}\left(U^{n}\right)$ by

Received by the editors December 6, 1977 and, in revised form, January 23, 1978.

AMS (MOS) subject classifications (1970). Primary 32A30, 46J20, 46J30; Secondary 46J10, $46 \mathrm{~J} 15$.

Key words and phrases. Inner functions, maximal ideal space, bounded analytic functions, polydisc, unimodular, closed subalgebra, $L^{\infty}\left(T^{n}\right)$.

${ }^{1}$ This research was done under the supervision of Dr. P. S. Chee at the University of Malaya and will form part of a thesis to be submitted for examination for the Ph.D. degree. 


$$
F\left(z^{\prime}, z^{\prime \prime}\right)=F_{1}\left(z^{\prime}\right) \quad \text { for }\left(z^{\prime}, z^{\prime \prime}\right) \in U^{2} \times U^{n-2} \text {. }
$$

Then

(i) $3 / 5>\left|F^{*}\right|>2 / 5$ a.e. on $E$,

(ii) $11 / 10>\left|F^{*}\right|>9 / 10$ a.e. on $T^{n} \backslash E$,

where $E=E_{1} \times T^{n-2}$ is a compact, circular subset of $T^{n}$ with empty interior and $m_{n}(E)>0$.

It can now be shown that $\left[H^{\infty} / \Sigma_{n}\right]$ is a proper closed subalgebra of $L^{\infty}\left(T^{n}\right)$ for $n>1$.

THEOREM 2. There is an $F$ in $H^{\infty}\left(U^{n}\right)$ such that $F^{*}$ is invertible in $L^{\infty}\left(T^{n}\right)$ but is not invertible in $\left[H^{\infty} / \Sigma_{n}\right]$ for $n>1$.

Proof. With $F$ as defined after Theorem $1, F^{*}$ is clearly invertible in $L^{\infty}\left(T^{n}\right)$ as it is bounded away from 0 . Now for any $f \in H^{\infty}\left(U^{n}\right), I \in \Sigma_{n}$, suppose that

$$
\left|\frac{1}{F^{*}}-\frac{f^{*}}{I^{*}}\right|<\frac{1}{9} \quad \text { a.e. on } T^{n}
$$

then

$$
\left|\frac{1}{F^{*}}\right|-\frac{1}{9}<\left|\frac{f^{*}}{I^{*}}\right|=\left|f^{*}\right|<\left|\frac{1}{F^{*}}\right|+\frac{1}{9} .
$$

Hence

$$
\left|f^{*}\right|>14 / 9 \text { a.e. on } E, \quad\left|f^{*}\right|<11 / 9 \text { a.e. on } T^{n} \backslash E \text {. }
$$

Since $E$ is circular,

$$
G>14 / 9 \text { a.e. on } E, \quad G<11 / 9 \text { a.e. on } T^{n} \backslash E,
$$

where $G(w)=\operatorname{ess} \sup _{|\alpha|=1}|f(\alpha w)|$. Suppose that $G=\psi$ almost everywhere and $\psi$ is lower semicontinuous. Then

$$
V=\left\{w \in T^{n}: \psi(w)>12 / 9\right\}
$$

is open and nonempty. But this is a contradiction as $G \neq \psi$ on $V \backslash E$ which is open and nonempty. Hence by a result of Rudin [3, Theorem 3.5.2], $f \notin H^{\infty}\left(U^{n}\right)$. This contradiction shows that

$$
\operatorname{dist}\left(\frac{1}{F^{*}}, \frac{H^{\infty}}{\Sigma_{n}}\right) \geqslant \frac{1}{9}>0 .
$$

Let $M_{n}$ and $X_{n}$ be the maximal ideal space of $H^{\infty}\left(U^{n}\right)$ and $L^{\infty}\left(T^{n}\right)$ respectively and define $\tau: X_{n} \rightarrow M_{n}$ by mapping each complex homomorphism of $L^{\infty}\left(T^{n}\right)$ to its restriction on $H^{\infty}\left(U^{n}\right)$. $\tau X_{n}$ is then the image of the maximal ideal space of $L^{\infty}\left(T^{n}\right)$ in $M_{n}$. The proof of the lemma in [1, p. 317] can be used to show that for $n>1$ too, the maximal ideal space $M\left[H^{\infty} / \Sigma_{n}\right]$ of $\left[H^{\infty} / \Sigma_{n}\right]$ can be identified with $K_{\Sigma_{n}}$, where

$$
K_{\Sigma_{n}}=\left\{\Phi \in M_{n}:|\Phi(I)|=1 \text { for all } I \in \Sigma_{n}\right\} \text {. }
$$

Range [2] has shown that the Shilov boundary $\partial_{n}$ of $H^{\infty}\left(U^{n}\right)$ is a proper 
subset of $\tau X_{n}$ for $n>1$. From Theorem 2, it can now be shown that again, unlike the case of the unit disc, $\tau X_{n} \neq K_{\Sigma_{n}}$.

THEOREM 3. For $n>, 1$

$$
\tau X_{n} \neq M\left[H^{\infty} / \Sigma_{n}\right]=K_{\Sigma_{n}}
$$

Proof. With $F$ as above, $F^{*}$ generates a maximal ideal in $\left[H^{\infty} / \Sigma_{n}\right]$ as it is not invertible. The corresponding complex homomorphism cannot belong to $\tau X_{n}$ since $F^{*}$ is invertible in $L^{\infty}\left(T^{n}\right)$.

\section{REFERENCES}

1. R. G. Douglas and W. Rudin, Approximation by inner functions, Pacific J. Math. 31 (1969), 313-320.

2. M. Range, $A$ small boundary for $H^{\infty}$ on the polydisc, Proc. Amer. Math. Soc. 32 (1972), 253-255.

3. W. Rudin, Function theory in polydiscs, Benjamin, New York, 1969.

Department of Mathematics, Univeristy of Malaya, Kuala Lumpur 22-11, Malaysia 